\title{
Coordinated observations of the quiet Sun transition region using SUMER spectra, TRACE images and MDI magnetograms
}

\author{
D. E. Innes*
}

\author{
Max-Planck-Institut für Aeronomie, Max-Planck-Str. 2, 37191 Katlenburg-Lindau, Germany \\ Received 1 June 2001 / Accepted 20 August 2001
}

\begin{abstract}
The relationship between the transition region ultraviolet emission and the underlying magnetic field is explored through a detailed analysis of SUMER Si IV line profiles, TRACE C IV, continuum and $171 \AA$ intensities, and MDI high resolution magnetic field measurements. The observations track a narrow area of quiet Sun near disk center over a period of 3 hours. Highly variable emission is found throughout. The most dramatic line Doppler shift and brightness variations come from a region of complex field evolution. The brightenings sometimes have faint elongated extensions in the TRACE CIV images. In most events with high Doppler shifts, the Si IV line wing reveals plasma flow 1-2 min before the line core brightens which suggests that plasma acceleration precedes plasma compression and/or heating. Simultaneous measurement of transition region densities using O IV line ratios imply large density fluctuations in the transition region plasma. There is an indication of waves of compression and rarefaction crossing the spectrometer's field-of-view. The waves' speed across the solar surface is between 20-40 $\mathrm{km} \mathrm{s}^{-1}$, and the compression may be as high as 10. In space-time images density fluctuations are much more prevalent and conspicuous than brightenings. In several cases the waves seem to start at the position of Si IV brightenings.
\end{abstract}

Key words. Sun: magnetic fields, transition region - UV radiation

\section{Introduction}

Rapid small-scale intensity variations dominate the dynamics of the solar transition region (Brueckner \& Bartoe 1983; Porter \& Dere 1991; Moses et al. 1994; Harrison 1997; Innes et al. 1997a). Ultraviolet emission lines such as Si IV, C IV and O VI often show factor $2-5$ brightness variations and Doppler shifts of up to $250 \mathrm{~km} \mathrm{~s}^{-1}$ (Brueckner \& Bartoe 1983; Dere et al. 1989). These events, known as micro-flares and explosive events, seem to be mostly confined to transition region temperatures. They are generally found above complex weak magnetic fields or on the edge of unipolar fields, rather than between simple bipole fields (Porter \& Dere 1991). Magnetic reconnection seems to be a viable energy source but there is some controversy about where, between photosphere and corona, the reconnection sites are.

Dere et al. (1991) and Innes et al. (1997b) suggest that reconnection in the upper photosphere produces the observed high velocity plasma flows directly. Another alternative, favored by Benz \& Krucker (1999) after analyzing radio and coronal ultraviolet emission of the quiet Sun, is that a primary energy release in the corona generates high energy particles that then heat and accelerate the chromospheric plasma below. Recently, Tarbell et al. (2000) and Ryutova \& Tarbell (2000) argue that the photosphere is

\footnotetext{
* e-mail: innes@linmpi.mpg.de
}

the primary energy input site and that reconnection here produces many shocks which interact and generate the observed flows in the transition region.

Much of the problem derives from the complex structure of the photosphere - corona interface. The transition region consists of many ever evolving loop-like structures embedded in a fine background of unresolved $2 \times 10^{4} \mathrm{~K}$ to $2 \times 10^{5} \mathrm{~K}$ plasma (Feldman et al. 1999, 2000). The main structures are related to small mixed polarity photospheric magnetic fields. The emission correlates well with the photospheric field strength but the loop-like structures do not, as one might expect, connect opposite polarity fields but appear to be either short, $5^{\prime \prime}$ bands, straddling across network lanes or longer, $20^{\prime \prime}$ to $30^{\prime \prime}$ wispy ribbons connecting a unipolar lane field to a point in the cell center (Warren \& Winebarger 2000). In general, the structures do not seem to extend either to the corona nor do they have bright foot-points in the chromosphere (Feldman et al. 1999).

It is against this background of loops and unresolved structures that transition region intensity and lineshift variations have to be considered. Any input of energy causing transition region lineshifts greater than $50 \mathrm{~km} \mathrm{~s}^{-1}$ will most probably be associated with a shock. Shocks may travel through the region causing plasma several thousands of kilometers from the original input site to be heated, compressed and accelerated. The structure of the 
shock-excited plasma, seen as brightenings, is expected to reflect the loop-like or elongated structures seen in transition region images. The observed line profiles will be determined by the angle that the shocks hit the loop-like structures and to which depth plasma acceleration occurs. To date, there are no quantitative models of the emission from shocked transition region loop structure. One can imagine that the emission will look elongated and if a shock hits a loop from the side the plasma may be accelerated both up and down the loop. Bi-directional flow is one of the recognized characteristics of transition region explosive events (Innes et al. 1997b).

Bi-directional flow out of a small energy input site is also one of the principle signatures of Petschek reconnection (Petschek 1964; Priest \& Forbes 1986). Based on the Petschek model and a measurement of the plasma flow speed and density, Dere et al. (1991) derived a magnetic field strength $20 \mathrm{G}$ in the reconnecting plasma. This is typical of the measured MDI flux in canceling photospheric fields but probably much less than the actual photospheric flux in the network and internetwork (Lin 1995). Numerical simulations of Petschek reconnection appropriate to the transition region (Innes \& Tóth 1999) demonstrated that the observed multi-component high velocity flows are possible but the simulations were not able to reproduce the observed line brightening at low velocity.

The aim of the observations presented here is to identify signatures of the brightenings that may be used in future models of the transition region energetics. This first requires an understanding of the basic emission and magnetic field properties of the observed region. So, after describing the observing sequences and data analysis techniques, we present an overview of the region. Correlations of brightenings and lineshifts to the field evolution are then shown in Sect. 4, where we also present the evidence for density fluctuations. Details of individual events are given in the last of the sections describing the observations. Our summary gives a few brief comments on what these observations seem to indicate.

\section{Data analysis}

An area of quiet Sun centered at solar coordinates $\left(-200^{\prime \prime}\right.$, $\left.250^{\prime \prime}\right)$ was observed for a period of 3 hours with SUMER (Wilhelm et al. 1995, 1997; Lemaire et al. 1997), TRACE (Handy et al. 1999) and MDI (Scherrer et al. 1995) on 19 June 1998.

The SUMER ultraviolet spectrometer was used to obtain stigmatic images of the SiIV and O IV lines near $1400 \AA$ with a cadence of 15 s from the same $1^{\prime \prime} \times 240^{\prime \prime}$ narrow section of the Sun throughout the period. Only the 50 pixels centered on the SiIV $1393.7 \AA$ line and the 100 pixels around the density sensitive O IV pair at 1399.8 $\AA$ and 1401.2 $\AA$ were transmitted. The $1^{\prime \prime} \times 300^{\prime \prime}$ slit was used but to meet telemetry constraints only the central $240^{\prime \prime}$ after binning by 2 could be transmitted. This resulted in a pixel size of $2^{\prime \prime}$ along the slit. Before binning the on board flat-field was applied. The flat-field had been taken 4 months earlier and this rather out-ofdate flat-field introduces some uncertainty in the derived line intensities and a somewhat larger uncertainty in the line ratios. In intensity, the uncertainty in the flat-field is of the order $5 \%$. This corresponds to a O IV line ratio uncertainty of about $10 \%$, and a density uncertainty of a factor 3. Because there was some danger that the Si IV line from the observed region of quiet Sun would have a count rate higher than 10 counts $\mathrm{px}^{-1} \mathrm{~s}^{-1}$ on some of the detector pixels, we followed the SUMER operation guideline and moved the spectral pixel address of the lines roughly every hour during the observing sequence. A detailed description of the commands to do this is given in Wilhelm et al. (1995). When the spectral pixel address of the line changes, the flat-field correction also changes because the line falls on a different part of the detector. During the three hour observing period the lines' position on the detector was moved three times and this causes jumps in the time evolution of the computed density (Sect. 3.3). The other important correction applied to the SUMER data was the geometric correction. As described in Wilhelm et al. (1997), this converts the raw "inverse cushion" images to "rectangular grid" images with lines straight to within one spectral and spatial pixel. Again, different geometric corrections at different pixel addresses causes jumps in the derived density. The spatial positions with steep intensity gradients are most sensitive to geometric correction errors.

TRACE observations were obtained with the $171 \AA$, $1550 \AA, 1600 \AA$, and $1700 \AA$ filters at full resolution (pixel size $\left.0.5^{\prime \prime}\right)$. Two sequences, one with the $171 \AA$ and one without $171 \AA$, were alternated. At times there are high cadence, every 15 s images in $1550 \AA, 1600 \AA$, and $1700 \AA$. For other periods the $171 \AA$ is included and then the cadence drops to $60 \mathrm{~s}$. Images in C IV were obtained using a linear combination of $1550 \AA, 1600 \AA$, and $1700 \AA$ intensities (Handy et al. 1999). The C IV outlines the transition region structures and brightenings. The main line in the $171 \AA$ filter is the Fe Ix line which has a formation temperature of $\sim 10^{6} \mathrm{~K}$.

The MDI provided high resolution magnetograms every $60 \mathrm{~s}$ with a pixel size of $0.5^{\prime \prime}$. The magnetograms shown in Sect. 5 are 5 min running averages with a final cut-off at $5 \mathrm{G}$ in order to bring out the more persistent magnetic field elements.

Images were first coaligned to within $5^{\prime \prime}$ using the given coordinates and pixel sizes in the image headers. Then, within that range, we adjusted the overlay for the best correspondence of the emissions. The TRACE images required magnification by $1 \%$ to allow for the $\mathrm{SOHO}-$ Sun and Earth-Sun distance difference. Then the TRACE $1700 \AA$ images could be cross-correlated with the high resolution MDI data closest in time. The TRACE $1550 \AA$ and $171 \AA$ images were then corrected for the spatial image offsets relative to the $1700 \AA$ images. The SUMER Si IV data were coaligned to the TRACE C IV images. 


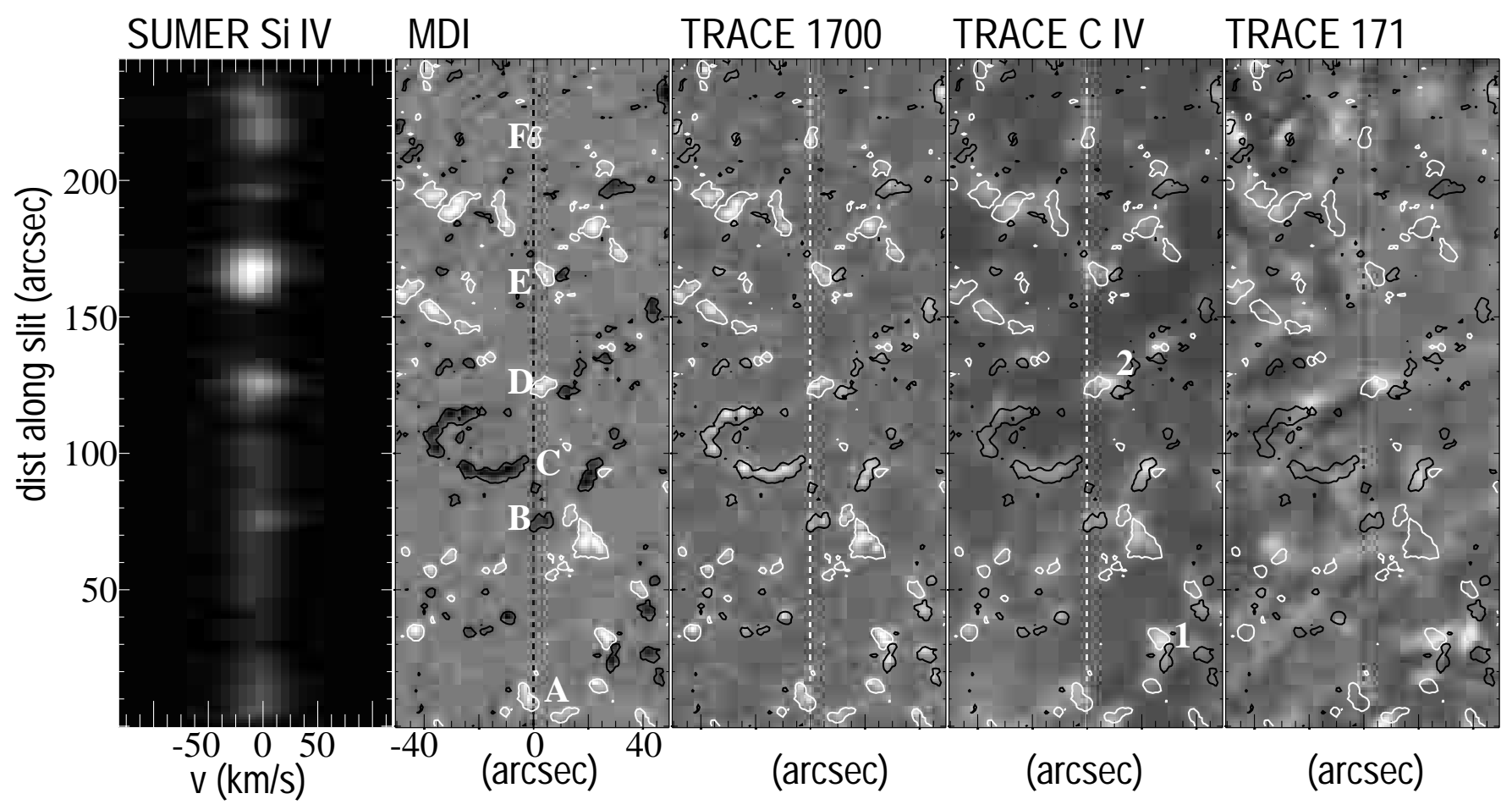

Fig. 1. Time-averaged images of the SUMER Si IV line, the MDI magnetic field and TRACE intensities. In the MDI and TRACE images, the dotted line running down the image center marks the position of the SUMER slit. The white (black) contours outline the $+(-) 9 \mathrm{G}$ fields. The positions of stronger magnetic field, labeled A-F along the SUMER slit in the MDI image, are also marked in Fig. 2. The brightest $\mathrm{C}$ IV and $171 \AA$ regions, e.g., 1 and 2 in the TRACE C IV image, lie between magnetic bipoles.

The TRACE images have had intensity spikes caused by high energy particles hitting the CCD removed. This was done by first identifying events and the effected pixels by looking for brightenings that occurred in just one of a time series of images. If a brightening was determined to be due to a high energy particle, interpolation in time was used to patch the dataset. In the C IV images, we had to look for both single image brightenings and dark spots because they were constructed with unfiltered continuum and $1550 \AA$ images. The individual $171 \AA$ images, taken with an exposure time $10 \mathrm{~s}$, are very noisy. Only one of the brightenings seen with SUMER, had corresponding $171 \AA$ data of sufficient quality to see the event. We have therefore decided to concentrate on the time-averaged appearance of the $171 \AA$ emission and its relationship to lower temperature activity and magnetic field rather than individual $171 \AA$ brightenings. About $300^{\prime \prime}$ to the west of the observed region was an active region. The active region emission caused a visible gradient in $171 \AA$ intensity across the observed area of quiet Sun. This has been removed from the $171 \AA$ images by dividing by the smoothed time-averaged image.

\section{Overview}

\subsection{Space}

The area observed is shown in Fig. 1. This is a typical region of quiet Sun with mixed polarity fields and many small-scale bright structures seen in the TRACE images.
The area contained several magnetic bipoles and predominantly unipolar regions. The bright points in the continuum image coincide with regions of high magnetic flux. The C IV is bright near the regions of high field. The very brightest $\mathrm{C}$ IV patches (marked 1 and 2) are between magnetic bipoles. The regions of bright $171 \AA$ (Fe IX) nearly always lie near regions of high fields. As noted by Falconer et al. (1998), the brightest regions of coronal emission are in general between bipoles.

The field-of-view of the SUMER crosses just east of two apparently bipolar regions (marked D and $\mathrm{E}$ in the MDI image) and over several that look basically unipolar (marked A, B, C, and F). At each position along the SUMER slit that is near a region of contoured magnetic flux, the Si IV intensity is bright against the darker background. During the three hours of observations the two regions, $\mathrm{D}$ and $\mathrm{E}$, that look like bipoles in the time-averaged MDI image, behaved quite differently. The lower one, D, was bright between the two poles in both C IV and $171 \AA$ throughout most the three hours. One could speculate, especially as the emission pattern is elongated from one pole to the other, that the field is a simple dipole. The emission from E, was more variable and produced simultaneous brightenings both between the poles and along its eastern edge (within the SUMER field-of-view). The combined effect is seen as a general brightening in the CIV time-averaged image. As discussed in Sect. 4.1 the photospheric field at $\mathrm{E}$ is more complex than a simple bipole.

The time-averaged Si IV line profiles (Fig. 1 left) also hint at different dynamics for the bipoles D and E. 


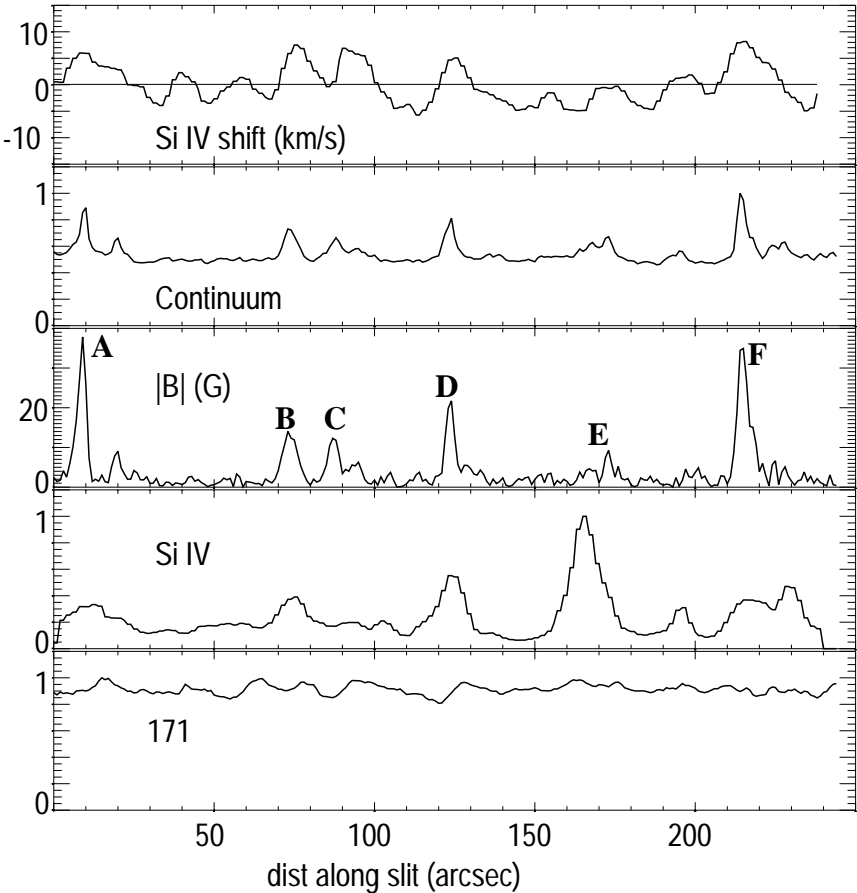

Fig. 2. Time-averaged quantities along the spectrometer slit showing the relationship between Si IV lineshift, $1700 \AA$ continuum, magnetic field strength, Si IV and $171 \AA$ intensities. The lineshifts are measured relative to the average lineshift along the slit and positive velocities correspond to redshifts. Intensities have been normalized to their maximum intensity. The stronger magnetic field regions, $\mathrm{A}-\mathrm{F}$, correspond to those labelled in Fig. 1.

The emission from the brightest bipole, E, is shifted more to the blue and appears more symmetric than that from D. This is illustrated more clearly in Fig. 2 where the time-averaged variation in lineshift, emission intensities and magnetic field are plotted along the slit. All the regions with relatively strong average field $(>10 \mathrm{G})$ are red-shifted. This includes bipole $\mathrm{D}$. The time average field at $\mathrm{E}$ is not strong and the line is slightly blue-shifted like in the internetwork or darker, low field regions. Previous analyses of transition region lineshifts have shown an average shift of $5 \mathrm{~km} \mathrm{~s}^{-1}$ at disk center (Peter \& Judge 1999) with typically spatial and temporal variations in lineshift of the order $\pm 10 \mathrm{~km} \mathrm{~s}^{-1}$ (Judge et al. 1997). If the average lineshift in Fig. 2 is at $5 \mathrm{~km} \mathrm{~s}^{-1}$ relative to the photosphere, then in the internetwork and near the peak of the Si IV intensity, the mean shift is zero. This implies there is no preferred up or down direction outside the regions of stronger magnetic field.

\subsection{Line profiles}

The average Si IV line profile is not a single Gaussian. In general the line, like the S VI line (Chae et al. 1998) can be best fit by two Gaussians consisting of a narrow central component and a second broader component to fit the wings. In Fig. 3, the time-average profiles along the slit, together with the difference from one and two Gaussian fits
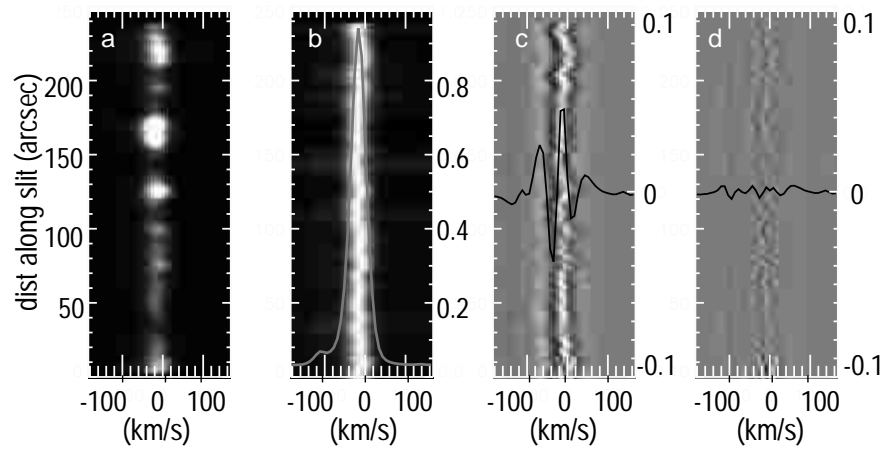

Fig. 3. Time-averaged Si IV a) line intensities, b) normalized line intensities with space-averaged profile over-plotted, c) difference between the normalized profiles and best single Gaussian fit with space-averaged over-plotted, and d) difference between normalized profiles and double Gaussian fit. The weak line near $-110 \mathrm{~km} \mathrm{~s}^{-1}$ has been fitted with a single Gaussian and subtracted from profiles in $\mathbf{c}$ ) and $\mathbf{d}$ ).

are shown. At every position along the slit, network and internetwork, the observed line profiles are best represented by a bright narrow core component and a weaker broad component that reproduces the wings. The line widths are close to those given in Chae et al. (1998) for S vi. The average narrow core Doppler width after correction for instrumental broadening is $21 \mathrm{~km} \mathrm{~s}^{-1}$. The wing component has an average Doppler width of $42 \mathrm{~km} \mathrm{~s}^{-1}$. Thus it seems that although the narrow core has a different shift in the network and internetwork, there is always an underlying broad component.

\subsection{Density}

The ratio of the observed OIV lines can, in principle, be used to determine the density of the transition region plasma (Judge et al. 1998; Pérez et al. 1999). Unfortunately the weaker of the density sensitive O IV line pair, at $1399 \AA$, is about a factor 20 weaker than the Si IV $1393 \AA$ line. It is not possible to obtain reliable O IV intensities and hence densities with the high space and time resolution used to make the observations. Therefore the O IV intensities used to compute the density have been obtained by binning the data in time and space.

In Fig. 4, the time-averaged line intensities and the corresponding line ratios are plotted. To further improve the signal-to-noise the emission has also been smoothed over $10^{\prime \prime}$. The line intensities have been computed by first subtracting a linear background and then fitting a single Gaussian to the line. The main uncertainties in the computed line ratio come from the accuracy of the fit, uncertainties in the background continuum, the flat-field and spatial coalignment of the two lines. The background continuum is computed, as described by Judge et al. (1998), using two windows. One window is short-ward of $1399 \AA$ and the other is long-ward of the $1401.5 \AA$ line. At all positions, the continuum at these two wavelengths is well defined and the uncertainties in the values used to compute the continuum level is smaller than the uncertainty in the 

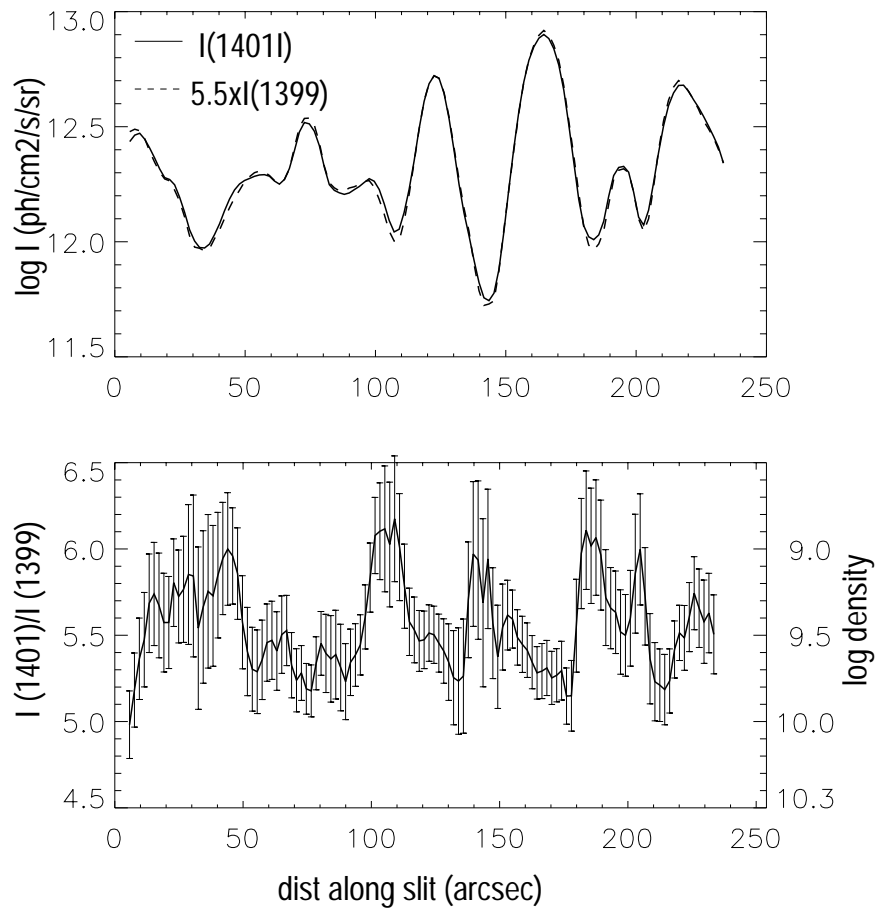

Fig. 4. Time-averaged intensity of the density sensitive O IV line pair along the spectrometer slit. In the top frame the intensity of the weaker line, multiplied by 5.5 , has been superimposed on the $\lambda 1401$ intensity. This has been done in order to help diagnose subtle inaccuracies in the geometric correction which may cause non-physical gradients in the density (lower frame) across the edge of network structures (e.g. between $125-140^{\prime \prime}, 190-200^{\prime \prime}, \sim 230^{\prime \prime}$ ).

line fit. The plotted error bars are combined background and line fit uncertainties. The misalignment uncertainties are more difficult to estimate. These data have been geometrically corrected but there may be small, $\sim 0.2$ pixel, misalignment at certain positions along the slit. Any bad alignment will show up as line ratio gradients across the edge of structures where there are sharp changes in intensity such as the network-internetwork boundaries. This is mostly not the case but the gradient around $200^{\prime \prime}$ and the dip at $230^{\prime \prime}$ may be partially due to misalignment. Flatfield errors can cause fairly significant errors in line ratios. The difference may be only $5 \%$ in intensity but it can accumulate to a factor 3 in density. During this observing sequence the detector grating was placed at four different positions. Therefore by averaging line ratios over the full sequence, the effect of flat-field errors may be reduced.

In general, lower densities (higher $I(1401) / I(1399)$ ratios) are in the lower intensity internetwork regions. Here the densities are of the order $10^{9} \mathrm{~cm}^{-3}$. The density in the network is about $4 \times 10^{9} \mathrm{~cm}^{-3}$ in agreement with previous authors (e.g., Griffiths et al. 1999).

\section{Evolution of network structures}

In this section, we show the temporal evolution of intensity and magnetic field within the SUMER field-of-view. Sites of variable intensity and events with high lineshift are identified. Later, the connection to the larger scale evolution of these structures will be presented.

\subsection{Magnetic field}

In the top two panels of Fig. 5, the TRACE CIV and continuum images are shown with the magnetic field contours overlayed. The correlation between the continuum and magnetic field is very obvious in the top right-hand frame (Fig. 5b). The C IV emission is strong both along the regions of stronger unipolar fields and along the mixed polarity lane around $170^{\prime \prime}$. The brightening along this lane is more clearly seen in the panel below. The lower panels show the time evolution of the SUMER SiIV and continuum intensities with the corresponding TRACE CIV and $1700 \AA$ continuum contours overlayed. The SUMER continuum is the average intensity $2 \AA$ to the red of the Si IV line. There is good correlation in both time and space between the observations of the two instruments in both line and continuum. The differences in the SUMER and TRACE intensities can be attributed to the difference in spatial and spectral resolutions of the two instruments. The SUMER Si IV image shows greater contrast between the very bright network lanes and the dark internetwork regions. The TRACE C IV shows 3 min oscillations but they are expected to be weak in the transition zone (Wikstøl et al. 2000), so there may be contamination of the TRACE C IV image by other emissions in the filter. The TRACE continuum, due to its better resolution and and higher count rate, shows much sharper definition of the network lanes than the SUMER continuum image. In all images the brightest intensities are from the five network lanes crossed by the spectrometer slit. The Si IV and to some extent the $\mathrm{C}$ IV has the brightest emission on the lane with the lowest field. During the observation period many weak, opposite polarity fields were seen to come and go below the slit.

\subsection{Explosive events}

There is no strict definition of an explosive event. They are small-scale $(\sim 4000 \mathrm{~km})$ dynamic events that have high velocity $\left(\sim 100 \mathrm{~km} \mathrm{~s}^{-1}\right)$ wings in their line profiles. Explosive events have been found both in the network and the internetwork. They are sometimes associated with brightenings and sometimes not. Chae et al. (2000) found that explosive events, defined as those events with non-thermal width greater than $45 \mathrm{~km} \mathrm{~s}^{-1}$, tend to occur on the edge and simultaneous with strong brightenings. We follow Winebarger et al. (1999) and use a criterion based on the flux in the line wing rather than just the non-thermal line width, to find events. In Fig. 6a, we identify events as brightenings and/or explosive events. The brightening contours (green) outline positions where the local (in time and space) Si IV intensity $(I)$ brightens above the background according to the formula $I / \operatorname{smooth}(I, 7) \geq 1.8$, where the background is the intensity smoothed with a 

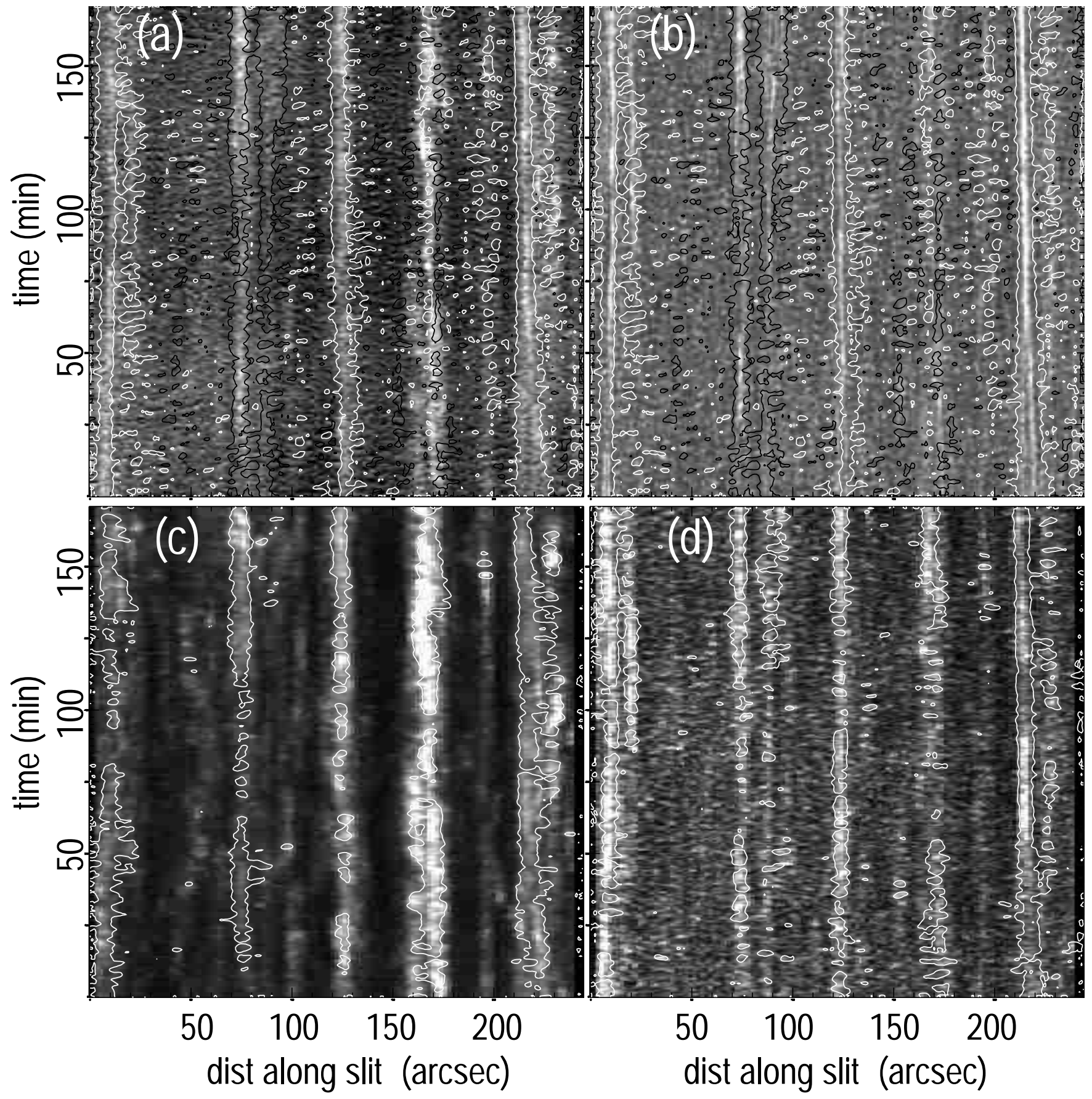

Fig. 5. Time evolution of emission and magnetic field along the spectrometer slit. a) TRACE CIV with MDI magnetic field white (black) contours at $+(-) 15$ G. b) TRACE $1700 \AA$ with MDI contours. c) SUMER Si IV with TRACE CIV contours. d) SUMER $1400 \AA$ continuum with TRACE $1700 \AA$ contours.

boxcar average of 7 space and 7 time pixels. The red and blue lines in Fig. 6a outline events where the intensity in the red or blue non-Gaussian wing is greater than $8 \times 10^{12} \mathrm{ph} \mathrm{s}^{-1} \mathrm{~cm}^{-2} \mathrm{sr}^{-1}$. The core Gaussian is assumed to have a Doppler width equal to the average width of the core component in Sect. 3.2 (i.e. $21 \mathrm{~km} \mathrm{~s}^{-1}$ ). With these criteria, there seem to be more brightenings than explosive events. About half the explosive events appear to be simultaneous in space and time with a brightening. Events with no associated brightening in this picture are often near a TRACE C IV brightening, just outside the SUMER field-of-view (e.g. E5, Fig. 12). The largest explosive events (and the largest brightenings) are found between $160^{\prime \prime}-180^{\prime \prime}$, on the edge of bipole E. The events marked E1 to E5 are discussed in more detail in the next section. 
(a)

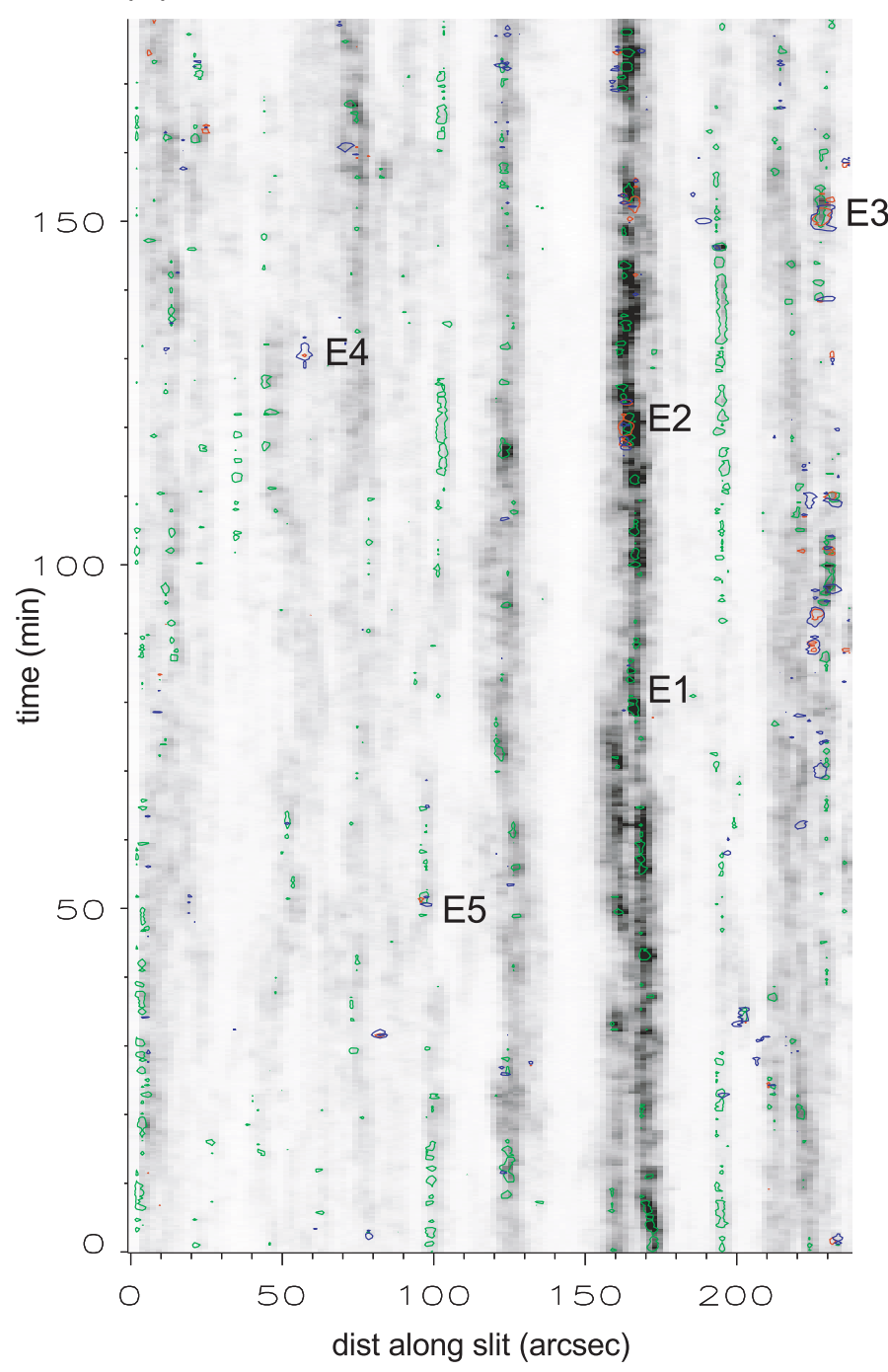

(b)

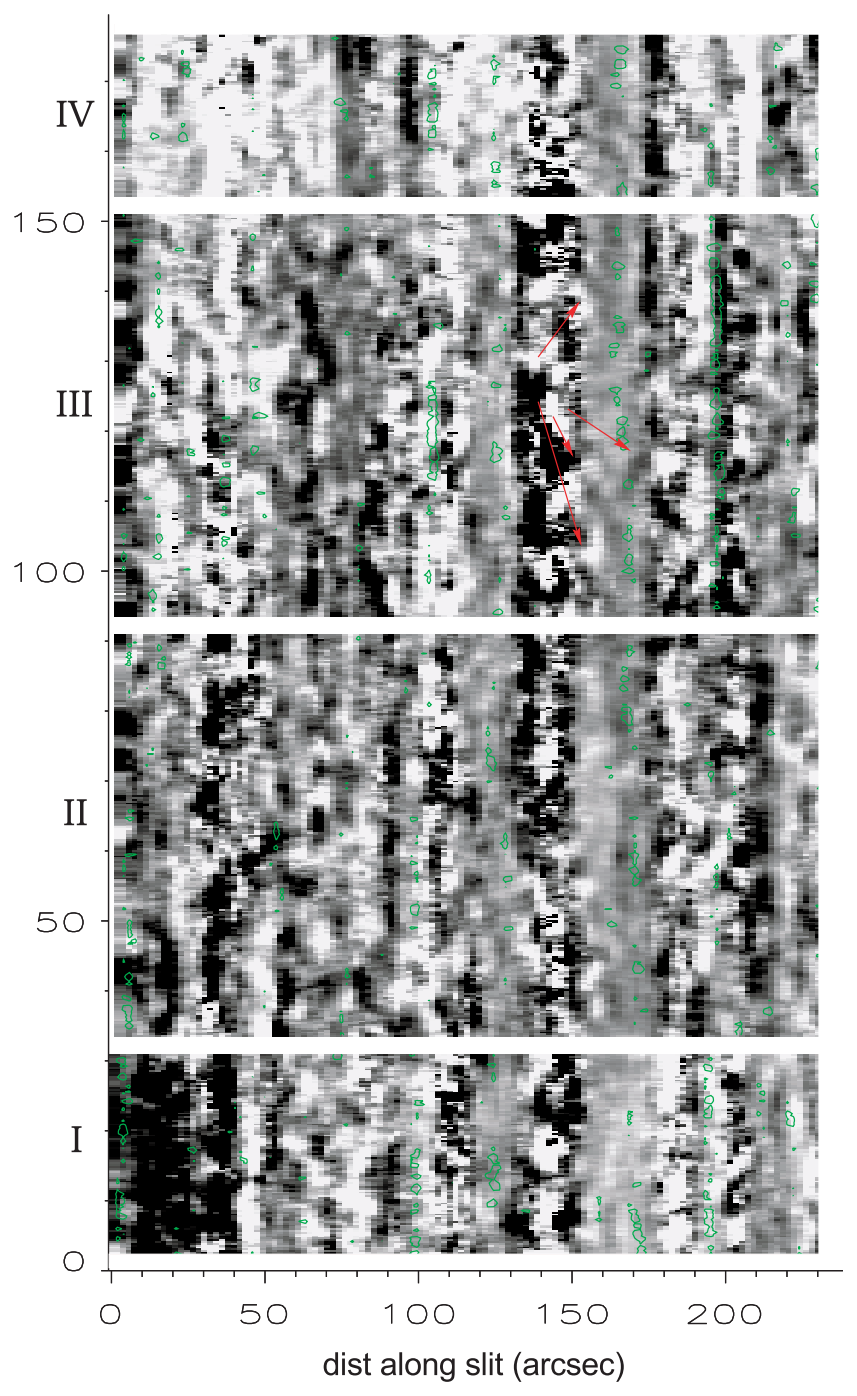

Fig. 6. a) Si IV intensity (negative) with green contours outlining the local brightenings and blue (red) contours events with significant emission in the blue (red) non-Gaussian wings of the line (see Sect. 4.2 for the criterion used). Individual events marked E1-E5 are discussed in Sect. 5. b) Density variations derived from O IV line ratios. The density scale ranges from $2 \times 10^{9}$ (white) to $3 \times 10^{10} \mathrm{~cm}^{-3}$ (black). Red arrows point to moving compression fronts (dark diagonal streaks). The green contours outline the local brightenings. The results for different detector positions (flat-field and geometric correction) have been separated by broad white bands and marked by block number on the left.

\subsection{Density variations}

Obtaining densities from O IV lines is rife with problems. The lines are weak so in most regions the signal-to-noise is low unless the integration time and/or the observed area is much larger than the event. In order to try and assess real from spurious effects and to bring out interesting relationships, we have integrated the data at several different resolutions. As mentioned in Sect. 3.3, the detector flat-field and the geometric correction also introduce significant uncertainties in the computed line ratios.

In Fig. $6 \mathrm{~b}$, the time variation of the density along the slit is presented alongside an image of the intensity variation. This ratio image is made using a spatial running average over $6^{\prime \prime}$ and a temporal running average over $2.75 \mathrm{~min}$. The image has been split into blocks with the same detector (flat-field and geometric correction) positions. Errors in the detector parameters show up as distinct trends within a block. For example, it is most probably an inappropriate flat-field that leads to obviously higher densities in block I than in block IV, between $0-40^{\prime \prime}$. The detector parameters should not influence time variations within a block.

The appearance of Fig. $6 \mathrm{~b}$ is generally speckled black and white patches indicating factor 10 density variations, within short time and space periods. The effect is strongest in the internetwork but there are also significant variations in the network. At the boundaries between the network and internetwork there is often a dark vertical line indicating longer lived high density transition region material. This could be an effect of the geometric correction but the fact that there are moments with no dark stripe for 

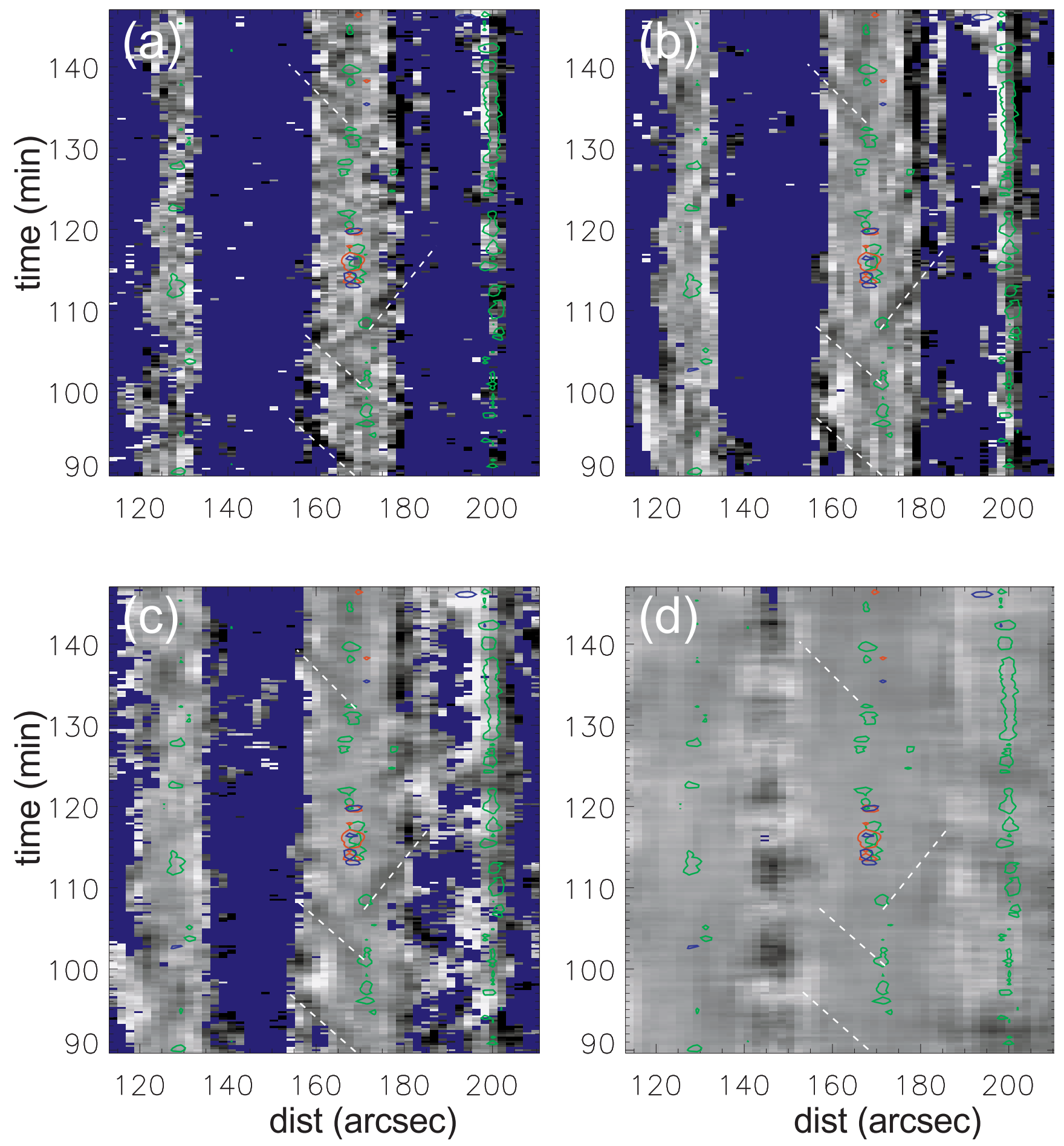

Fig. 7. Density variations computed at different space and time resolutions a) 1.25 min and $2^{\prime \prime}$ b) 2.75 min and $2^{\prime \prime}$ c) 2.75 min and $6^{\prime \prime}$ d) $5.25 \mathrm{~min}$ and $10^{\prime \prime}$. The positions of the brightenings and explosive events are outlined with green and blue and red contours. Data points where the uncertainty in the line intensity is greater than $20 \%$ are colored blue. The density scale ranges from $2 \times 10^{9}$ (white) to $3 \times 10^{10} \mathrm{~cm}^{-3}$ (black). In each frame, white dashed lines trace the position of possible compression fronts. The fronts were identified in c) and then the lines were re-drawn on the other frames at the same positions.

the same detector position suggests that the effect may be real. Perhaps it is related to the larger than average redshift seen by Judge et al. (1997) (their Fig. 2) along the network-internetwork boundaries. Another feature, seen best in the network between slit positions $155^{\prime \prime}-180^{\prime \prime}$ are the dark diagonal streaks indicated by red arrows in block III.

Dark diagonal streaks in the density image with no corresponding intensity increase could be moving compression fronts. The angles of the streaks give front speeds 
along the detector slit of between $20-40 \mathrm{~km} \mathrm{~s}^{-1}$. The compression is often greater than 4 which is the compression behind a strong shock. Moving fronts with this high compression are therefore most likely shocks. In which case it appears that shocks are continuously crossing and interacting with the transition region plasma.

Although it is clear that large density fluctuations do occur in the transition region, there is considerable uncertainly as to whether the fronts are real or not. We have tried numerous techniques to determine the most accurate values of the $\mathrm{O}$ IV line ratios and in every case come across the problem of low signal-to-noise. In Fig. 7, we show the O IV line ratios computed at different resolutions. Points with an uncertainty in the O IV line intensities greater than $20 \%$ are colored blue. The contours marking the brightenings and the explosive events from Fig. 6a have been added.

In Fig. 7c, the dark diagonal streaks show up best. As examples we have identified four with dashed white lines. All four start at the position of a brightening. It is interesting that there is no density increase associated with the large explosive event at the position where the red, blue and green contours overlap.

The lowest resolution uses a very course, $5.25 \mathrm{~min}$ and $10^{\prime \prime}$, averaging. This brings out the large density fluctuations in the internetwork. It looks as though the 810 min period density fluctuations seen by Pérez et al. (1999), are present in this dataset as well.

\section{Individual events}

The events, marked E1 to E5 in Fig. 7, have been selected to illustrate how different regions of the network reacted during the observing sequence. Each highlighted event has quite different $\mathrm{C}$ IV structure but each event is also typical for its underlying field. The general magnetic field does not change much from that shown in Fig. 1 during the three hours although small field elements seem to appear and disappear. Likewise the basic event characteristics at any one position do not change much although there are large brightness variations.

There are two positions along the slit where the majority of the explosive events occur. One, between $160^{\prime \prime}-170^{\prime \prime}$ marked E in Fig. 1, is on the eastern edge of a magnetic bipole. In Fig. 5, we can see that this is a region of ever changing mixed polarity fields. The other explosive event area is very near the north of the slit near $220^{\prime \prime}$. Again Fig. 5 shows signs of mixed polarity fields but not any more than at, for example, $100^{\prime \prime}$ or $190^{\prime \prime}$ both of which produced a series of brightenings.

The first event E1 is shown in Fig. 8. This is a simple brightening event on the edge of bipole E in Fig. 1 The line brightens by a factor of about 2 and then fades within $2 \mathrm{~min}$. The TRACE images and contours on the MDI images show that the brightening lies between the positive pole of the bipole and a small region of negative flux on its NE edge. These images also show simultaneous brightening on the bipole neutral line about $10^{\prime \prime}$ to the west and

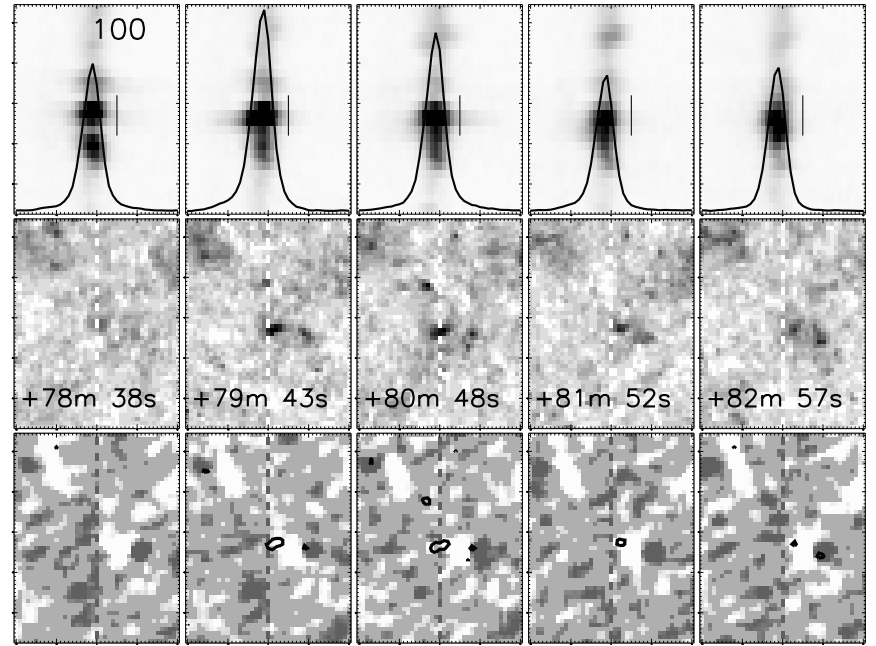

Fig. 8. Evolution of event E1. In the top row are the SUMER stigmatic images (negative) with the line profile from the section indicated by the short black vertical line overplotted. The velocity scale runs from blue shifts of $200 \mathrm{~km} \mathrm{~s}^{-1}$ on the left to red shifts of $200 \mathrm{~km} \mathrm{~s}^{-1}$. The maximum of the intensity is at $100 \mathrm{cts}^{-1} \mathrm{px}^{-1}\left(1 \mathrm{cts}^{-1} \mathrm{px}^{-1}=7.7 \times\right.$ $\left.10^{12} \mathrm{ph} \mathrm{s}^{-1} \mathrm{~cm}^{-2} \mathrm{sr}^{-1} \AA^{-1}\right)$. The second row is the corresponding TRACE C IV image (negative) with the time from the beginning of the observation run marked. The third row is the corresponding MDI image with TRACE CIV contours overlaid. Magnetograms span from $-10 \mathrm{G}$ (black) to $10 \mathrm{G}$ (white). The images are centered around $170^{\prime \prime}$ from the bottom of the slit and cover an area $40^{\prime \prime} \times 55^{\prime \prime}$. The dashed lines down the center of the MDI and TRACE images mark the position of the SUMER slit.

at a point about $15^{\prime \prime}$ north, just east of the SUMER fieldof-view, with no obvious related magnetic field structures. This northern point brightens in C IV several times during the 3 hours observation period. It occurs on one of the regions of enhanced $171 \AA$ emission in the time-averaged image (Fig. 1), so there is probably some coronal structure at this position.

The event E2 (Fig. 9), from the same region as E1, was the strongest explosive event in the observing period. The velocities extended beyond $150 \mathrm{~km} \mathrm{~s}^{-1}$ to both the blue and red. Plasma acceleration occurred at the start of the event and it did not reach maximum brightness until 3 min later, near the end of the event. Nearly all events seen in this sequence and by Ryutova \& Tarbell (2000) follow a similar pattern: first the lineshifts and then 1-2 min later, maximum brightness. The TRACE images for this event show several interesting features. The most obvious is that, like the event E1, there is an associated $\mathrm{C}$ IV brightening lying along the neutral line of the main bipole $10^{\prime \prime}$ to the west. More subtle is the small brightening on the NE edge of the positive pole that is connected to the position of the main event by a faint loop-like structure in the images at $+115 \mathrm{~m} 20 \mathrm{~s}$.

The next event E3 (Fig. 10) also started with shifts and then brightened at line center. It lasted about $6 \mathrm{~min}$. At the start of the event there were no noticeable fields. 


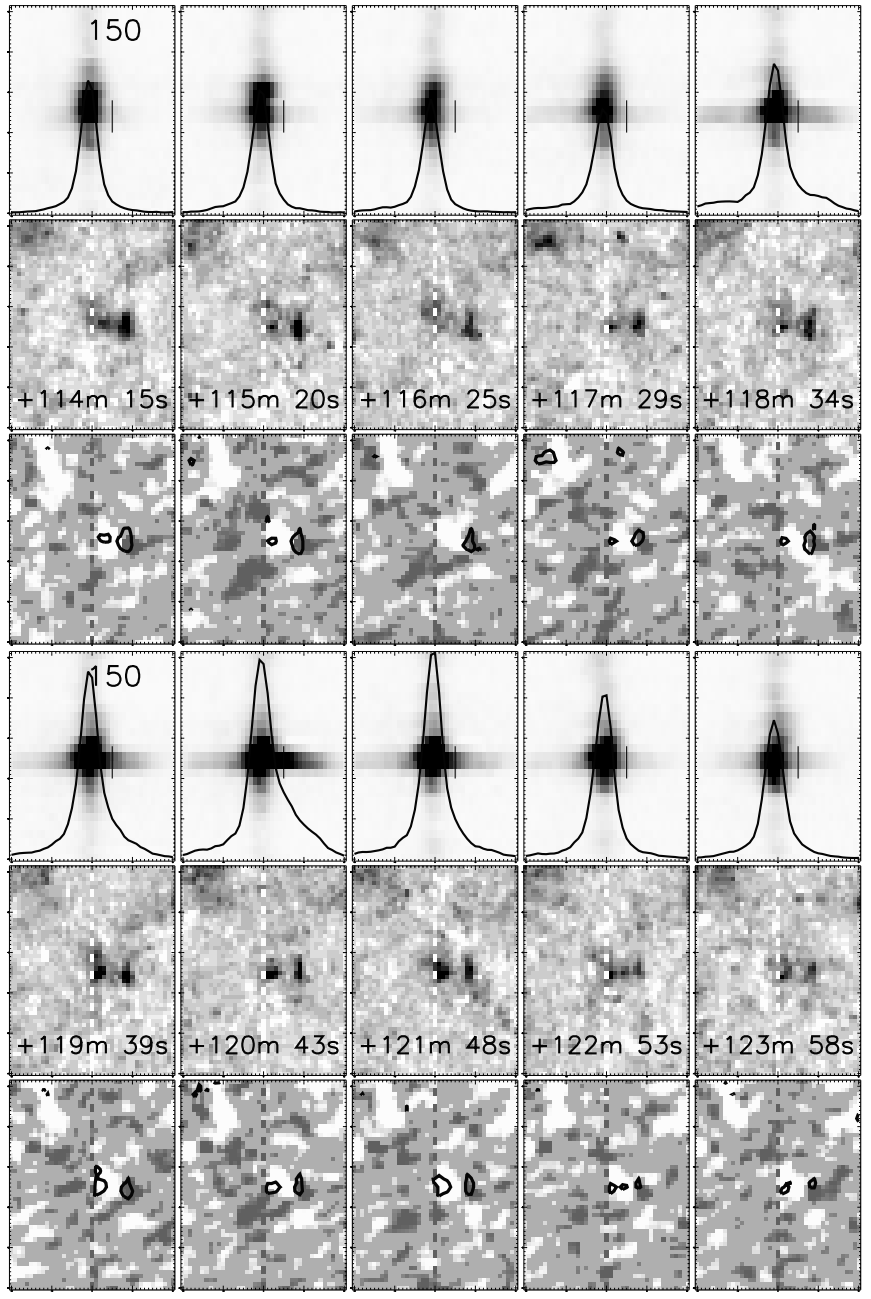

Fig. 9. Evolution of event E2 laid out as above. This event is brighter than E1 and the maximum of the Si IV intensity scale is at $150 \mathrm{ct} \mathrm{s}^{-1} \mathrm{px}^{-1}$.

At the end of the event there were small weak positive and negative fields. There is simultaneous C IV brightening along the neutral line of the small bipole $15^{\prime \prime}$ south of the explosive event. The main brightening is $3-4^{\prime \prime}$ east of the slit and there are no strong shifts at the SUMER slit position.

The event E4 (Fig. 11) is an example of an isolated event that seems to occur in the cell center. This position has low magnetic field and continuum and C IV intensity. The $171 \AA$ intensity in Fig. 1 is enhanced at exactly this position. This suggests hot coronal plasma at this position with no obvious associated photospheric fields. In this case the brightening at line center and the high Doppler shifts occur simultaneously.

The fifth event (Fig. 12) is a typical example of an explosive event on the edge of a unipolar field region. Doppler shifts of the order $50 \mathrm{~km} \mathrm{~s}^{-1}$ accompany line center brightening and the event lasts about 1-2 min. The main C IV brightening is just east of the SUMER slit at the end of the event. The images in Fig. 12 are also interesting because they show the structure and shifts associated

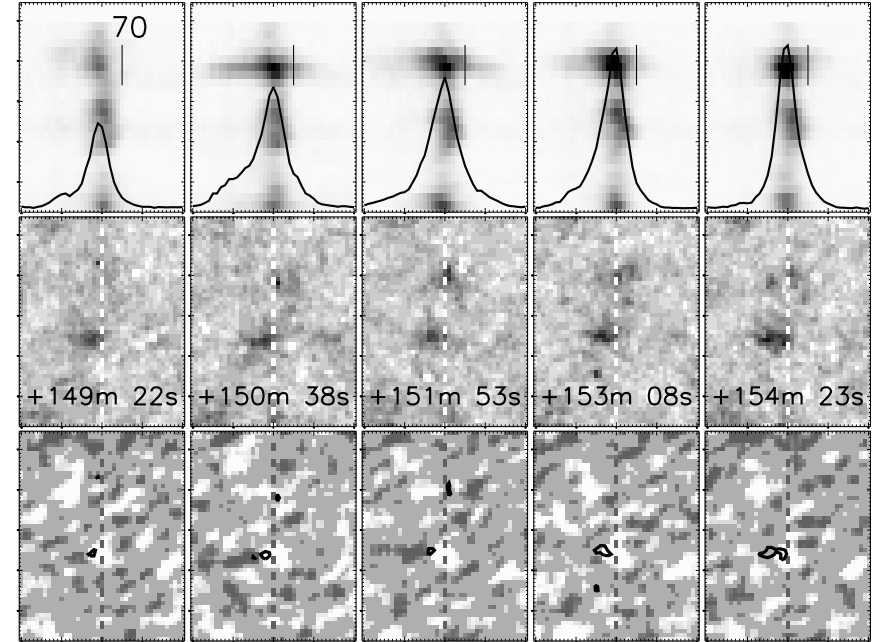

Fig. 10. Evolution of event E3 as above. The maximum of the Si IV intensity scale is at $70 \mathrm{ct} \mathrm{s}^{-1} \mathrm{px}^{-1}$ and the image center at $220^{\prime \prime}$ along the slit.

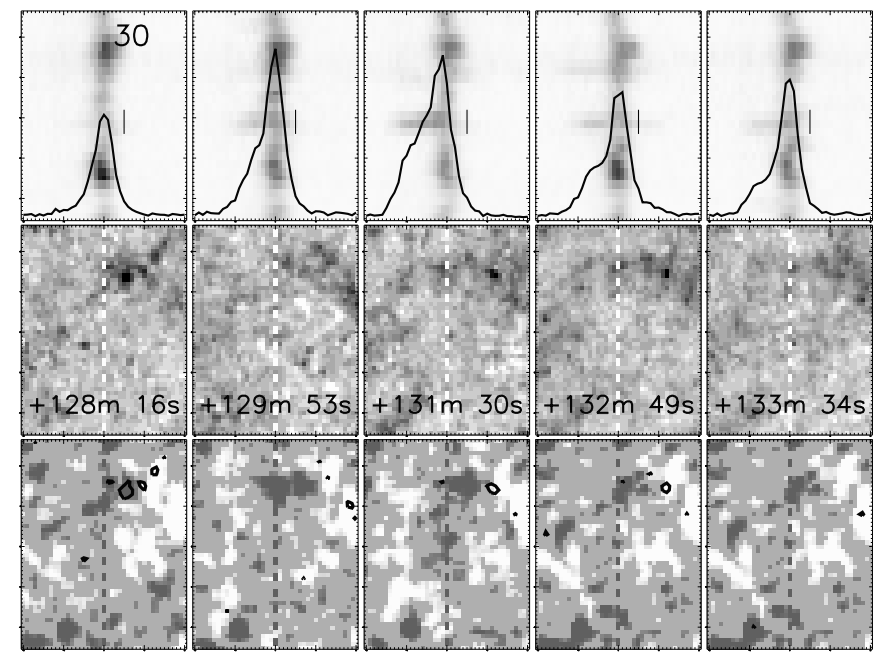

Fig. 11. Evolution of event E4 as above. The maximum of the Si IV intensity scale is at $30 \mathrm{ct} \mathrm{s}^{-1} \mathrm{px}^{-1}$ and the image center at $60^{\prime \prime}$ along the slit.

with a brightening in the "less active" bipole (D in Fig. 1). The C IV structure runs from one pole to the other. There are only small shifts at the position of the spectrometer slit along the eastern edge of the bipole region.

\section{Summary}

The dynamics of a small section of quiet Sun have been observed over a period of three hours. This series of observations confirm that the sites showing most transition region variability and the highest lineshifts are those that overlie complex photospheric field configurations. The active sites continued to evolve and produce high velocity plasma flows and brightenings throughout the three hours. Some transition region brightenings were accompanied by small (6-9 $\left.\operatorname{arcsec}^{2}\right)$, short-lived (4-10 min) coronal brightenings. 


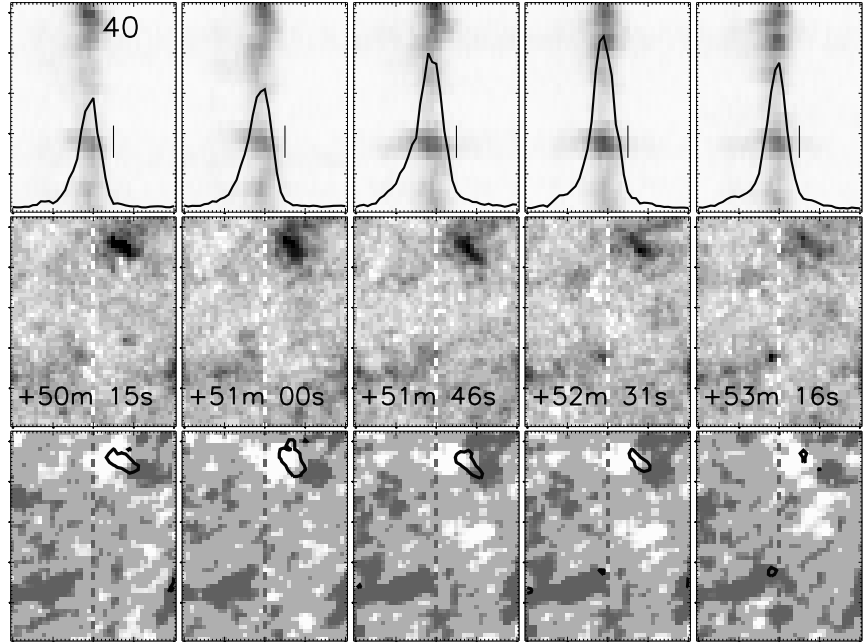

Fig. 12. Evolution of event E5 as above. The maximum of the Si IV intensity scale is at $40 \mathrm{ct} \mathrm{s}^{-1} \mathrm{px}^{-1}$ and the image center at $110^{\prime \prime}$ along the slit.

Sometimes the C IV brightenings had an elongated or jet-like structure. The brightenings, their position and orientation with respect to the underlying field tend to repeat. For example, the structure associated with the complex region, E in Fig. 1, continually produces multiple brightenings. Nearly always the brightest emission is between centers of opposite polarity field. The strongest explosive event seen in this sequence came from this region. It is associated with a jet-like brightening in the TRACE C IV image, lying along the neutral line of the bipole. In most explosive events the line wings brightened 1-2 min before the line core indicating that some of the plasma is first accelerated before the rest of the plasma is heated and/or compressed.

Simple bipole and/or unipolar fields are associated with less dramatic brightening in the transition region. Again the brightening may have an elongated structure. In the example shown (Fig. 12) the long axis seems to connect more persistent fields of opposite polarity. Above regions of stronger photospheric fields, lineshifts are smaller and the average flow is directed downward towards the photosphere.

Various factors seem to support the idea of shocked loop-like structures. The first is that the O IV data suggest propagating density enhancements in the transition region plasma. If shocks are traveling across and through the transition region, one would expect the average line profile to have a broad second component, both locally near the brightening sites and in the dark internetwork. This seems to be the case for our data although it is not seen in other datasets (Peter 2000). Finally the brightenings repeat at the edge of strong field regions which is the position of the transition region loop-like structures (Warren \& Winebarger 2000).

Acknowledgements. I would like to thank Ted Tarbell for his help with the TRACE observing sequences and data reduction and Klaus Wilhelm and Nick Hoekzema for constructive comments. The SUMER project is financially supported by DARA, CNES, NASA and the ESA PRODEX programme (Swiss contribution). SUMER and MDI are part of SOHO, the Solar and Heliospheric Observatory of ESA and NASA.

\section{References}

Benz, A. O., \& Krucker, S. 1999, A\&A, 341, 286

Brueckner, G. E., \& Bartoe, J. F. 1983, ApJ, 272, 329

Chae, J., Schühle, U., \& Lemaire, P. 1998, ApJ, 505, 957

Chae, J., Wang, H., Goode, P. R., Fludra, A., \& Schühle, U. 2000, ApJ, 528, L119

Dere, K. P., Bartoe, J.-D. F., \& Brueckner, G. E. 1989, Sol. Phys., 123, 41

Dere, K. P., Bartoe, J. F., Brueckner, G. E., Ewing, J., \& Lund, P. 1991, J. Geophys. Res., 96, 9399

Falconer, D. A., Moore, R. L., Porter, J. G., \& Hathaway, D. H. 1998, ApJ, 501, 386

Feldman, U., Dammasch, I. E., \& Wilhelm, K. 2000, Space Sci. Rev., 93, 411

Feldman, U., Widing, K. G., \& Warren, H. P. 1999, ApJ, 522, 1133

Griffiths, N. W., Fisher, G. H., Woods, D. T., \& Siegmund, O. H. W. 1999, ApJ, 512, 992

Handy, B. N., Acton, L. W., Kankelborg, C. C., et al. 1999, Sol. Phys., 187, 229

Harrison, R. A. 1997, Sol. Phys., 175, 467

Innes, D. E., Brekke, P., Germerott, D., \& Wilhelm, K. 1997a, Sol. Phys., 175, 341

Innes, D. E., Inhester, B., Axford, W. I., \& Wilhelm, K. 1997b, Nature, 386, 811

Innes, D. E., \& Tóth, G. 1999, Sol. Phys., 185, 127

Judge, P., Carlsson, M., \& Wilhelm, K. 1997, ApJ, 490, L195

Judge, P. G., Hansteen, V., Wikstol, O., et al. 1998, ApJ, 502, 981

Lemaire, P., Wilhelm, K., Curdt, W., et al. 1997, Sol. Phys., 170,105

Lin, H. 1995, ApJ, 446, 421

Moses, D., Cook, J. W., Bartoe, J. F., et al. 1994, ApJ, 430, 913

Pérez, M. E., Doyle, J. G., O’Shea, E., \& Keenan, F. P. 1999, A\&A, 351, 1139

Peter, H. 2000, A\&A, 360, 761

Peter, H., \& Judge, P. G. 1999, ApJ, 522, 1148

Petschek, H. E. 1964, in AAS-NASA Symposium on the Physics of Solar Flares, ed. W. N. Hess, NASA-SP 50 (Washington DC: NASA), 425

Porter, J. G., \& Dere, K. P. 1991, ApJ, 370, 775

Priest, E. R., \& Forbes, T. G. 1986, J. Geophys. Res., 91, 5579

Ryutova, M. P., \& Tarbell, T. D. 2000, ApJ, 541, L29

Scherrer, P. H., Bogart, R. S., Bush, R. I., et al. 1995, Sol. Phys., 162, 129

Tarbell, T. D., Ryutova, M., \& Shine, R. 2000, Sol. Phys., 193, 195

Warren, H. P., \& Winebarger, A. R. 2000, ApJ, 535, L63

Wikstøl, Ø., Hansteen, V. H., Carlsson, M., \& Judge, P. G. 2000, ApJ, 531, 1150

Wilhelm, K., Curdt, W., Marsch, E., et al. 1995, Sol. Phys., 162,189

Wilhelm, K., Lemaire, P., Curdt, W., et al. 1997, Sol. Phys., 170,75

Winebarger, A. R., Emslie, A. G., Mariska, J. T., \& Warren, H. P. 1999, ApJ, 526, 471 